\title{
Control of an active rectifier with an inductive-capacitive-inductive filter using a Twisting based algorithm
}

\author{
Arnau Dòria-Cerezo \\ Dept. Electrical Eng. and \\ Inst. Industrial and Control Eng. \\ Polytechnical University of Catalonia \\ 08028 Barcelona, Spain \\ Email: arnau.doria@upc.edu
}

\author{
Paul F. Puleston \\ CONICET and LEICI \\ National University of La Plata \\ CC91, 1900, La Plata, Argentina \\ Marie Curie Fellow, IRI, CSIC-UPC \\ 08028 Barcelona, Spain \\ Email: puleston@ing.unlp.edu.ar
}

\author{
Cristian Kunusch \\ Inst. de Robòtica i Informàtica Industrial \\ Consejo Superior de Inv. Científicas \\ Polytechnical University of Catalonia \\ 08028 Barcelona, Spain \\ Email: ckunusch@iri.upc.edu
}

\begin{abstract}
This paper presents a novel controller for an active rectifier with an inductive-capacitive-inductive filter. The proposed control scheme comprises two levels. The internal level, is a current controller based on the second order sliding mode Twisting algorithm, which robustly ensures an unity power factor at the connection point. The slower external level is a PI controller in charge of regulating the output DC voltage to a desired reference. The controller setup also includes a finite-time observer of the current derivative that can be used to avoid direct (and sometimes problematic) numerical differentiation. Finally, simulation results are presented to validate the proposed control scheme.
\end{abstract}

\section{INTRODUCTION}

The use of active rectifiers has beed widely increased in the last decades thanks to its ability to transform the AC electrical power to a DC power. The advantages of using these power converters are the power factor correction and its robustness to guarantee the DC bus voltage for a large range of load values. However, the commutation of the set of switches implies an undesired current ripple of the inductor filter. This effect can be considerably reduced using an inductive-capacitive-inductive (LCL) filter instead of the traditional first order inductance filter, and low total harmonic distortion (THD) of the current can be attained.

The key goal of a single-phase active rectifier is to ensure a given DC voltage with an unity power factor on the grid side. These objectives are usually fulfilled by using control algorithms that measure some of the variables (normally, voltage and current on the grid side and the DC voltage) and process that information to provide a suitable switching policy to reach the desired set points. Several control techniques, from classical proportional-integral-derivative (PID) controllers to more advanced nonlinear methodologies, have been applied to different power converter topologies. However, in the literature, mostly linear based controllers are used for active rectifiers with an LCL filter, see examples in [1][2] (classical PI controllers), [3][4] (deadbeat control), [5] (spacestate based PI controller), [6] (resonant PI control). The main disadvantages of using linear techniques are that the inherent nonlinear behavior of the full-bridge rectifier is disregarded and the results are valid only locally.

The use of control methodologies that rely on non-linear approaches allow a systematic design procedure of robust controllers, guaranteeing stability and performance in extended operation ranges. One non-linear control technique specially appropriate to switched power converters is the Sliding Mode Control (SMC) approach [7]. Due to the fact that the control action is commonly discontinuous, it is particularly suitable to deal with variable structure systems such as power converters [8], resulting in controlled dynamical systems with enhanced performance. However, one of the main problems when implementing sliding mode based controllers is that the switching frequency is variable. A possible solution to obtain a fixed switching frequency is to apply a control algorithm which synthesizes a continuous (or even discontinuous) control law and then process it by means of pulse-width modulation (PWM) devices.

In this framework, considering the system relative degree (RD) and specific design issues (variables differentiation, computational burden, etc.) a Twisting Algorithm (TA) is proposed to solve the rectifier control problem. This algorithm belongs to the so-called family of Second Order Sliding Mode (SOSM) controllers and is specially intended for relative degree two systems, providing finite time convergence and insensibility to coupled Lipschitz disturbances.

The main contribution of this paper is the development of a SOSM based control strategy for a single-phase active rectifier with an LCL filter. The control setup comprises two levels, namely a robust inner current control, designed using the Twisting Algorithm methodology, and an outer control level, in charge of regulating the DC voltage in the presence of load variations. Additionally, a uniform robust exact differentiator for the current error is included in order to avoid numerical problems during the implementation.

The paper is organized as follows: in the next section, the single-phase active rectifier is introduced, and its dynamical model is given. In Section III the whole control scheme is proposed. Then, Section IV presents the design of the current controller based on the TA, and also includes, the zero 


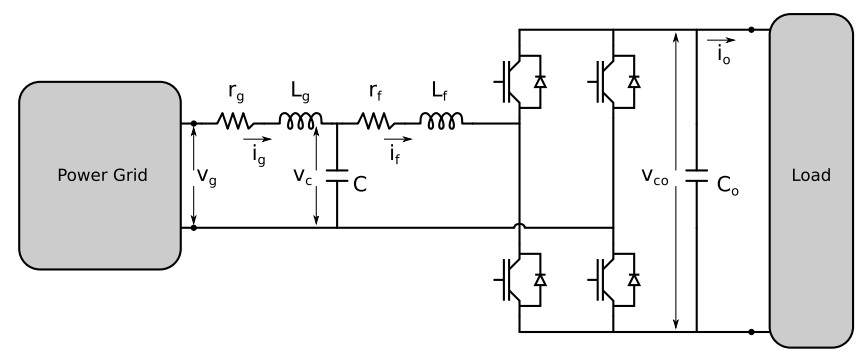

Fig. 1. Simplified scheme of a single-phase rectifier with a LCL filter.

dynamics analysis and the observer for the time derivative of the current error. In Section V the use of a PI controller for the regulation of the DC voltage is justified. Section VI shows the numerical simulations performed to validate and assess the performance of the proposed controller. Finally, the conclusions are stated in Section VII.

\section{MODEL OF A SINGLE-PHASE ACTIVE RECTIFIER WITH AN INDUCTIVE-CAPACITIVE-INDUCTIVE FILTER}

Figure 1 shows a simplified scheme of a single-phase rectifier with a LCL filter. The dynamics of this system is described by

$$
\begin{aligned}
L_{g} \frac{\mathrm{d} i_{g}}{\mathrm{~d} t} & =-r_{g} i_{g}-v_{C}+v_{g}, \\
C \frac{\mathrm{d} v_{C}}{\mathrm{~d} t} & =i_{g}-i_{f}, \\
L_{f} \frac{\mathrm{d} i_{f}}{\mathrm{~d} t} & =-r_{f} i_{f}+v_{C}-u v_{C o}, \\
C_{o} \frac{\mathrm{d} v_{C o}}{\mathrm{~d} t} & =u i_{f}-\frac{v_{C o}}{R},
\end{aligned}
$$

where $i_{g}, i_{f}$ are the input and output currents of the LCL filter, respectively, $v_{C}$ is the voltage in the filter capacitor, $C$, and $v_{C o}$ is the voltage in the DC link. The load has been considered a pure resistance, $R$. The power grid voltage is $v_{g}=V_{g} \sin (\omega t)$ (where $V_{g}$ and $\omega$ are the voltage amplitude and frequency, respectively) and $u \in[-1,1]$ is the control signal and represents the duty cycle of the set of switches. $L_{g}$ and $L_{f}$ are the LCL filter inductances, $C$ and $C_{o}$ are the capacitances in the LCL filter and DC link, respectively, and $r_{g}, r_{f}$ represent the inductor loses. Equations (1) and (4) can be rewritten in an affine in the control form as

$$
\dot{x}=f(x, t)+g(x, t) u(t),
$$

where $x^{T}=\left(i_{g}, v_{C}, i_{f}, v_{C_{o}}\right)$,

$$
f(x, t)=\left(\begin{array}{c}
-\frac{1}{L_{g}}\left(r_{g} i_{g}+v_{C}\right) \\
\frac{1}{C}\left(i_{g}-i_{f}\right) \\
\frac{1}{L_{f}}\left(v_{C}-r_{f} i_{f}\right) \\
-\frac{1}{R C_{o}} v_{C_{o}}
\end{array}\right)
$$

and

$$
g(x, t)=\left(\begin{array}{c}
0 \\
0 \\
-\frac{1}{L_{f}} v_{C_{o}} \\
\frac{1}{C_{o}} i_{f}
\end{array}\right) .
$$

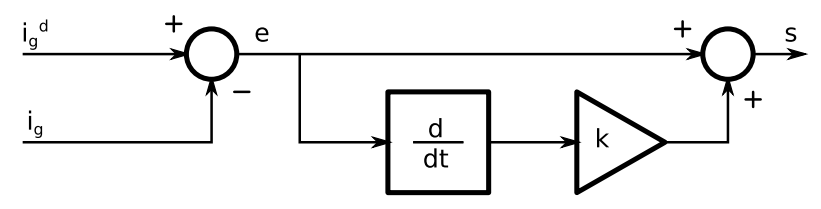

Fig. 3. Sliding variable block diagram.

\section{Control objectives AND OVERALl CONTROL SCHEME}

The control objective is twofold. To regulate the DC link voltage, $v_{C o}$, to a desired value $v_{C o}^{d}$ and to have unity power factor at the connection point. These two requirements can be simultaneously attained by tracking an input current reference of the form $i_{g}^{d}=I_{g} \sin (\omega t)$. The amplitude $I_{g}$ is then computed by the outer loop to maintain the DC voltage at its desired value, while the $\sin (\omega t)$ is obtained from the power grid voltage $v_{g}$.

Figure 2 shows a schematic representation of the proposed control setup, with the SOSM inner-loop controlling the gridside current $i_{g}$. For the outer control, a PI controller is used to regulate the DC voltage, $v_{C_{o}}$, to its reference, $v_{C_{0}}^{d}$. The control action generated by this external loop is, precisely, the required amplitude of the grid current, $I_{g}$, while the grid voltage carrier is obtained by means of a phase-locked loop (PLL). Then, the current reference $i_{g}^{d}$ is constructed by direct multiplication.

\section{SLIDING MODE CURRENT CONTROLLER DESIGN}

\section{A. Control objective in terms of SMC}

The first step for the design of the sliding mode controller is to define an sliding variable $s$ in terms of the current error $e=$ $i_{d}-i_{g}^{d}$, taking into account that the ultimate current objective is to track the grid current reference, ensuring robust convergence to $e=0$. Given that the error $e$ is $\mathrm{RD} 3$ with respect to $u$, it cannot be used as sliding variable by its own (note that classical SMC requires $s$ to be RD 1). However, advantage can be taken of SOSM techniques, which are capable to deal with relative degree 2 . Then, a simple RD 2 sliding variable can be defined just adding a derivative term

$$
s=e+k \dot{e},
$$

where $k>0$, is a design parameter. Notice that this SOSM approach needs only one time differentiator in $s$ (see Figure 3 ), while classical SMC would also required to include full information of the second time derivative into $s$, in order to attain relative degree 1 .

Moreover, the time derivative of the current reference required in (6) can be reconstructed using the PLL outputs $\cos (\omega t)$ and $\omega$. An schematic representation of the proposed computation of $s$ is depicted in Figure 4.

\section{B. Second order SMC design}

At this point, with the current control objective adequately embedded into $s$, a robust controller can be straightforwardly designed in the framework of second order sliding mode. Considering the system characteristics and the proposed RD 2 sliding variable, a suitable SOSM control algorithm that 


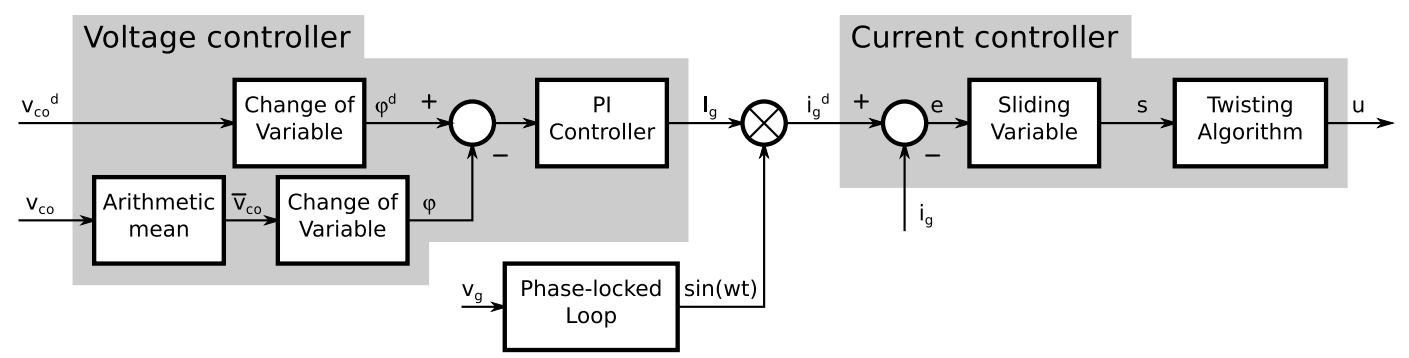

Fig. 2. Overall control scheme.

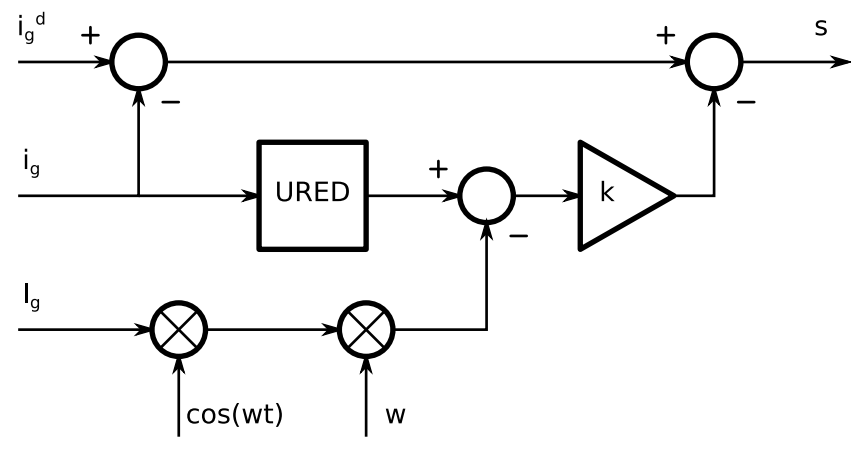

Fig. 4. Modified scheme to compute the sliding variable, $s$.

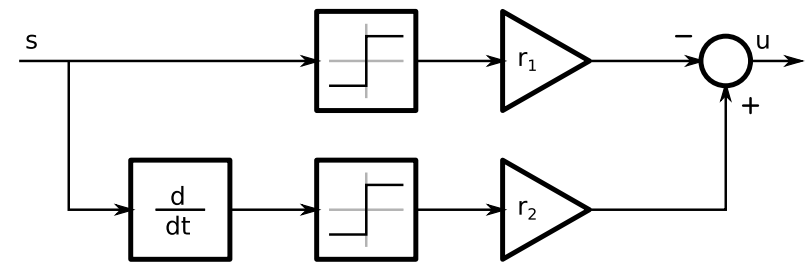

Fig. 5. Twisting Algorithm control scheme.

guarantees robust convergence to $s=0$ in finite time (and consequent fulfillment of the aforementioned current objective), is the so called Twisting Algorithm [9][10],

$$
u=-r_{1} \operatorname{sign}(s)-r_{2} \operatorname{sign}(\dot{s}) .
$$

Note that its implementation is considerably simple, requiring low on-line computational burden (see Fig. 5). It has only two constant parameters, $r_{1}$ and $r_{2}$, tuned in accordance with certain system bounds as stated in [10], which are obtained off-line during the design phase.

Specifically, given the sliding variable (6) and the affine in the control form (5) of the active rectifier, the second time derivative of $s$ can be written

$$
\ddot{s}=\varphi^{\prime}(x, t)+\gamma^{\prime}(x, t) u(t) .
$$

In a bounded region of operation $\varphi^{\prime}(x, t)$ and $\gamma^{\prime}(x, t)$ are uncertain (disturbed) but uniformly bounded functions, as follows

$$
0<\Gamma_{m}^{\prime} \leq \gamma^{\prime}(x, t) \leq \Gamma_{M}^{\prime}
$$

and

$$
\left|\varphi^{\prime}(x, t)\right| \leq \Phi^{\prime}
$$

The controller parameters $r_{1}$ and $r_{2}$ are tuned such that they simultaneously satisfy the following conditions

$$
\begin{gathered}
r_{1}>r_{2}>0 \\
\Gamma_{m}^{\prime}\left(r_{1}+r_{2}\right)-\Phi^{\prime}>\Gamma_{M}^{\prime}\left(r_{1}-r_{2}\right)+\Phi^{\prime} \\
\Gamma_{m}^{\prime}\left(r_{1}-r_{2}\right)>\Phi^{\prime} .
\end{gathered}
$$

Then, in the bounded region of operation, it is proved that the Twisting controller (7) generates a second order sliding mode that robustly attracts the trajectories of the system to $s=\dot{s}=0$ in finite time [10].

\section{Analysis of the zero dynamics}

The zero dynamics (or ideal sliding dynamics) can be interpreted as the dynamics that remains once the controller has forced the system to reach and stay on the manifold $s=\dot{s}=0$. Its behavior completes the analysis of the closed loop system defined by (5) with (7).

The constraint $s=0$ establishes the following algebraic dependence between $i_{g}$ and $v_{C}$,

$$
i_{g}=\frac{k v_{C}+\left(L_{g} I_{g}-k V_{g}\right) \sin (\omega t)+k \omega L_{g} I_{g} \cos (\omega t)}{L_{g}-k r_{g}} .
$$

Similarly, the second constraint $\dot{s}=0$, relates the dynamics of $i_{f}$ with $i_{g}$ and $v_{C}$, which together with (8) yields

$$
v_{C}=c_{1} i_{f}+c_{2} \sin (\omega t)+c_{3} \cos (\omega t)
$$

where $c_{1}, c_{2}$ and $c_{3}$ are (10), (11) and (12), respectively.

Finally, it is substituted the discontinuous control action $u$ with an equivalent control (i.e., a fictitious continuous control that would make the sliding surface flow invariant), which can be obtained from $\ddot{s}=0$ and takes the form

$$
u_{e q}=\frac{1}{d_{1} v_{C_{o}}}\left(d_{2} i_{f}+d_{3} \sin (\omega t)+d_{4} \cos (\omega t)\right),
$$

where $d_{1}, d_{2}, d_{3}$ and $d_{4}$ are given by (14), (15), (16) and (17), respectively.

Substituting $v_{C}$ and $u$ with (9) and (13) in the system (1)(4), the order of the closed loop dynamics is reduced in two. It results

$$
\begin{aligned}
L_{f} \frac{\mathrm{d} i_{f}}{\mathrm{~d} t}= & -\left(r_{f}-c_{1}+\frac{d_{2}}{d_{1}}\right) i_{f}+\left(c_{2}-\frac{d_{3}}{d_{1}}\right) \sin (\omega t) \\
& +\left(c_{3}-\frac{d_{4}}{d_{1}}\right) \cos (\omega t) \\
C_{o} \frac{\mathrm{d} v_{C o}}{\mathrm{~d} t}= & \frac{1}{d_{1}}\left(d_{2} i_{f}+d_{3} \sin (\omega t)+d_{4} \cos (\omega t)\right) \frac{i_{f}}{v_{C_{o}}} \\
& -\frac{v_{C o}}{R}
\end{aligned}
$$




$$
\begin{aligned}
c_{1} & =\frac{k\left(L_{g}-k r_{g}\right)}{k^{2}+C\left(L_{g}-k r_{g}\right)} \\
c_{2} & =\frac{\left(k L_{g} \omega^{2}-r_{g}\right) C\left(L_{g}-k r_{g}\right)-k L_{g}}{k^{2}+C\left(L_{g}-k r_{g}\right)} I_{g}+V_{g} \\
c_{3} & =\frac{\omega\left(C\left(k^{2} r_{g}^{2}-L_{g}^{2}\right)-k^{2} L_{g}\right)}{k^{2}+C\left(L_{g}-k r_{g}\right)} I_{g}+\frac{k C \omega\left(L_{g}-k r_{g}\right)}{k^{2}+C\left(L_{g}-k r_{g}\right)} V_{g} .
\end{aligned}
$$

$$
\begin{aligned}
d_{1}= & k^{3}+C\left(L_{g}-k r_{g}\right) k \\
d_{2}= & -\left(r_{f}+r_{g}\right) k^{3}+\left(r_{f} r_{g} C+L_{f}+L_{g}\right) k^{2}-C\left(L_{f} r_{g}+L_{g} r_{f}\right) k+L_{g} L_{f} C \\
d_{3}= & \left(r_{g} C \omega^{2}\left(L_{f}-L_{g}\right) k^{3}+\left(r_{g}^{2} C+C \omega^{2}\left(L_{g}\left(L_{g}+L_{f}\right)-r_{g}^{2} C L_{f}\right)-L_{f}-L_{g}\right) k^{2}+r_{g} C\left(L_{f}-L_{g}\right) k\right) I_{g} \\
& -\left(C L_{g} L_{f}\left(1-L_{g} C \omega^{2}\right)\right) I_{g}+\left(1-C L_{f} \omega^{2}\right)\left(k^{3}-r_{g} C k^{2}+L_{g} C k\right) V_{g} \\
d_{4}= & \left(\omega\left(C\left(L_{g} L_{f} \omega^{2}+r_{g}^{2}\right)-L_{f}-L_{g}\right) k^{3}-L_{g} \omega^{3} C^{2} L_{f} r_{g} k^{2}+C \omega\left(C L_{f}\left(L_{g}^{2} \omega^{2}+r_{g}^{2}\right)-L_{g}\left(L_{g}+L_{f}\right)\right) k-L_{g} r_{g} C^{2} L_{f} \omega\right) I_{g} \\
& +C \omega\left(-r_{g} k^{3}+\left(L_{f}+L_{g}\right) k^{2}-r_{g} C L_{f} k+L_{g} C L_{f}\right) V_{g} .
\end{aligned}
$$

The zero dynamics in (18) only depends on $i_{f}$. If $r_{f}-c_{1}+$ $\frac{d_{2}}{d_{1}}>0, i_{f}$ is stable with oscillations at frequency $\omega$. Then, the remaining dynamics of (19) can be seen as

$$
v_{C_{o}} C_{o} \frac{\mathrm{d} v_{C o}}{\mathrm{~d} t}=-\frac{v_{C o}^{2}}{R}+h(t),
$$

where $h(t)$, due to the product of $i_{f}(t)$ with sinusoidal functions, is a periodic function with frequency $2 \omega$. Assuming $v_{C_{o}}(t)>0$, it is transformed into the following linear ODE with the change of coordinates $\xi=\frac{1}{2} v_{C_{o}}^{2}$ (see [11] for further details)

$$
C_{o} \frac{\mathrm{d} \xi}{\mathrm{d} t}=-\frac{2}{R} \xi+h(t)
$$

with stable solutions for $C_{o}, R>0$. This analysis reveals that a perfect current tracking yields oscillations at frequency $2 \omega$ in the $v_{C_{o}}$ voltage, similar to the results presented in [11].

\section{Uniform robust exact differentiator}

The RD 2 expression of $s$ designed in (6) depends on the time derivative of the current $i_{g}$. However, in some actual cases, it is preferred to avoid the implementation of direct differentiators, among other reasons due to practical noise issues. Therefore, in this control proposal, the variable $\frac{d i_{g}}{d t}$ can be computed by means of an uniform robust exact differentiator (URED) [12], a time derivate observer based on the generalized super twisting algorithm reported in [13].

Let $\eta(t)$ be a Lebesgue-measurable function defined on $[0, \infty)$ and take it as the input signal. Suppose that $\eta(t)$ can be decomposed as

$$
\eta(t)=\eta_{0}(t)+v(t)
$$

where $\eta_{0}(t)$ is the unknown base signal to be differentiated and $v(t)$ corresponds to a uniformly bounded noise signal. Assuming that $\eta_{0}(t)$ is twice continuously differentiable and that the first derivative is Lipschitz with known Lipschitz constant $L>0$.

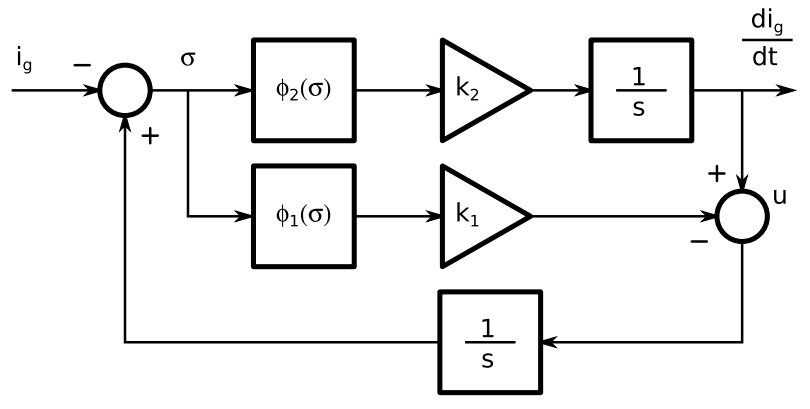

Fig. 6. Block diagram of the uniform robust exact differentiator.

Defining the error signal $\sigma=z_{0}-\eta$, the following dynamical system can be constructed

$$
\begin{aligned}
& \dot{z}_{0}=-k_{1} \phi_{1}(\sigma)+z_{1} \\
& \dot{z}_{1}=-k_{2} \phi_{2}(\sigma),
\end{aligned}
$$

where

$$
\begin{aligned}
& \phi_{1}(\sigma)=|\sigma|^{\frac{1}{2}} \operatorname{sign}(\sigma)+|\sigma|^{\frac{3}{2}} \operatorname{sign}(\sigma) \\
& \phi_{2}(\sigma)=\frac{1}{2} \operatorname{sign}(\sigma)+2 \sigma+\frac{3}{2} \mu^{2}|\sigma|^{2} \operatorname{sign}(\sigma),
\end{aligned}
$$

and $k_{1}$ and $k_{2}$ are constant positive gains dependent on $L$. The variable $z_{0}$ and $z_{1}$ are the estimations of $\eta_{0}$ and $\dot{\eta}_{0}$, respectively. Indeed, $z_{1}(t)$ converges exactly to $\dot{\eta}_{0}$ in finitetime, with the convergence time independent of the initial differentiation error (see [12] for details). See the block diagram implementation in Figure 6.

Note that an observer for $\frac{d i_{g}}{d t}$ based on the URED converges to its true value in finite-time. This is an important advantage, given that the current SOSM Twisting controller can be connected once the convergence time of the URED has elapsed and, consequently, undesirable errors due to the observer transient are avoided. 


\section{PI OUTER-LOOP VOLTAGE CONTROL}

The slower DC bus voltage control loop is designed assuming an ideal current tracking (i.e. $i_{g}=i_{g}^{d}$ ), which is in fact guaranteed in finite time. In Section IV-C, the zero dynamics was studied where $i_{g}^{d}=I_{g} \sin (\omega t)$ with $I_{g}$ constant. This analysis concluded that, in steady state, oscillations at $2 \omega$ frequency appeared in the DC bus voltage, $v_{C o}$. Consequently, the control objective should be turned into the regulation of the averaged value ${ }^{1}$ of the DC bus voltage, $\bar{v}_{C o}$, rather than of the instantaneous voltage $v_{C o}$.

Assuming a perfect current tracking, the control law designed in the previous subsection guarantees

$$
i_{g}=I_{g}(t) \sin (\omega t),
$$

where $I_{g}(t)$ is the required amplitude to regulate the DC bus voltage at the desired value. Replacing (20) in (1), (2), (3) and (4) one gets a complex dynamics with the oscillations predicted in Section IV-C and the (first, second and third) time derivative of $I_{g}(t)$. Then, averaging the resulting dynamics, with the following coordinates change

$$
\psi=\frac{1}{2} \bar{v}_{C o}^{2},
$$

and assuming slow variations of $I_{g}(t)$, one gets

$$
C_{o} \frac{\mathrm{d} \psi}{\mathrm{d} t}=-\frac{2}{R} \psi-a_{2} I_{g}^{2}+a_{1} I_{g}-a_{0},
$$

where $I_{g}$ can be used as a control action to regulate $\psi$ at a desired value $\psi^{d}=\frac{1}{2}\left(v_{C o}^{d}\right)^{2}$, and

$$
\begin{aligned}
a_{2} & =\frac{\pi\left(r_{f}\left(\omega^{2} L_{g} C-1\right)^{2}+\omega^{2} r_{g}^{2} r_{f} C^{2}+r_{g}\right)}{\omega}, \\
a_{1} & =\frac{\pi V_{g}\left(1+2 \omega^{2} r_{g} r_{f} C^{2}\right)}{\omega}, \\
a_{0} & =\pi V_{g}^{2} \omega r_{f} C^{2} .
\end{aligned}
$$

Notice that since all the parameters are positive, $a_{2}, a_{1}, a_{0}>0$.

Replacing the parameters of the rectifier used in the simulation stage (see Section VI),

$$
\begin{aligned}
& a_{2}=9.9 \cdot 10^{-5} \\
& a_{1}=3.11 \\
& a_{0}=3.8 \cdot 10^{-4}
\end{aligned}
$$

reveals that $a_{1}$ is clearly dominating $a_{2}$ and $a_{0}$, and (21) can be approximated by the linear system

$$
C_{o} \frac{\mathrm{d} \psi}{\mathrm{d} t}=-\frac{2}{R} \psi+a_{1} I_{g}
$$

which can be regulated by means of a PI controller

$$
I_{g}=k_{p}\left(\psi^{d}-\psi\right)+\int\left(\psi^{d}-\psi\right) \mathrm{d} t,
$$

with $k_{p}, k_{i}>0$ and $\psi^{d}=\frac{1}{2}\left(\bar{v}_{C_{o}}^{d}\right)^{2}$.

\footnotetext{
${ }^{1}$ The averaged function of a periodic signal $f(t)$ of period $T$ is calculated as $\bar{f}(t)=\frac{1}{T} \int_{t-T}^{t} f(\tau) \mathrm{d} \tau$.
}

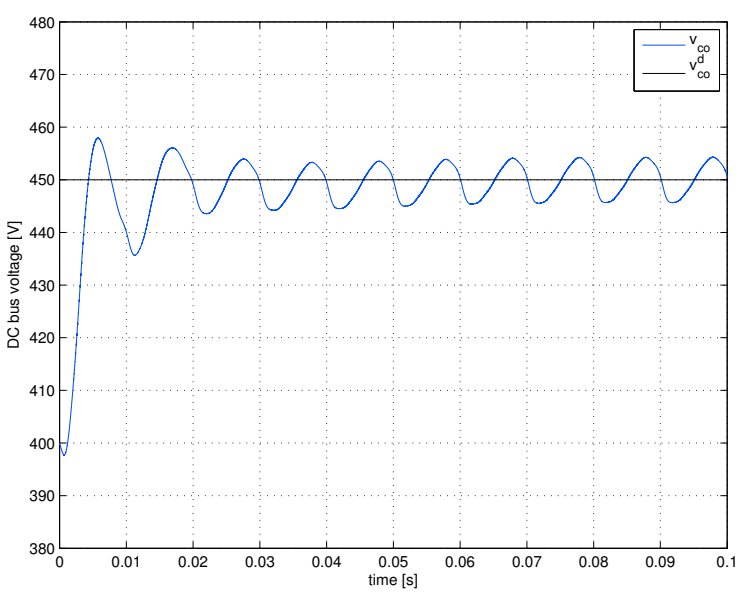

Fig. 7. Simulation results: DC bus voltage after a reference step change, from $400 \mathrm{~V}$ to $450 \mathrm{~V}$.

\section{Simulations}

In order to validate the control algorithm presented in this paper numerical simulations have been performed using a fixed time step of $5 \cdot 10^{-8} \mathrm{~s}$. The sliding variable has been implemented using the URED to observe the time derivative of $i_{g}$ and minimize possible numerical differentiation issues.

In this section two different simulation tests are shown. The first one consists in a change of the DC voltage reference, from $400 \mathrm{~V}$ to $450 \mathrm{~V}$, with a load of $R=50 \Omega$. The second test consists in a sudden change of the load form $R=50 \Omega$ to $R=20 \Omega$. The parameters of the power converter are: $L_{g}=$ $300 \mu \mathrm{H}, r_{g}=50 \mathrm{~m} \Omega, C=20 \mu \mathrm{F}, L_{f}=100 \mu \mathrm{H}, r_{f}=50 \mathrm{~m} \Omega$ and $C_{o}=2 \mathrm{mF}$. The control gains are: $k=10^{-4}, r_{1}=0.7$ and $r_{2}=0.3$ (for the Twisting Algorithm), and $k_{p}=0.75$ and $k_{i}=50$ (for the external PI controller).

In Figure 7, the DC voltage $v_{C_{o}}$ and its reference value $v_{C_{o}}^{d}$ are depicted. The voltage is regulated after a short transient time, even when the oscillations predicted in Section IV-C appear. Meanwhile, the grid current $i_{g}$ is in phase with the voltage grid $v_{g}$ as Figure 8 shows, implying an unity power factor. The results of the second test are shown in figures 9 and 10. In this case, the change of the load value implies a transient before the controller fulfills the control goals. Similarly to the previous case, the average of the DC voltage is regulated with an unity power factor.

\section{CONCLUSIONS AND FINAL REMARKS}

A general controller for an active rectifier with an inductive-capacitive-inductive filter was developed. Taking into account several features, such as the system relative degree, robustness and implementation simplicity, a non-linear control approach was proposed using a second order sliding mode Twisting algorithm in conjunction with an exact differentiator. This results in a robust and relatively simple control structure that particularly suits the current tracking problem. In addition, satisfactory zero dynamics analysis is provided for the current loop. The controller setup is completed with an outer loop based on a PI controller for the regulation of the average output DC voltage. 


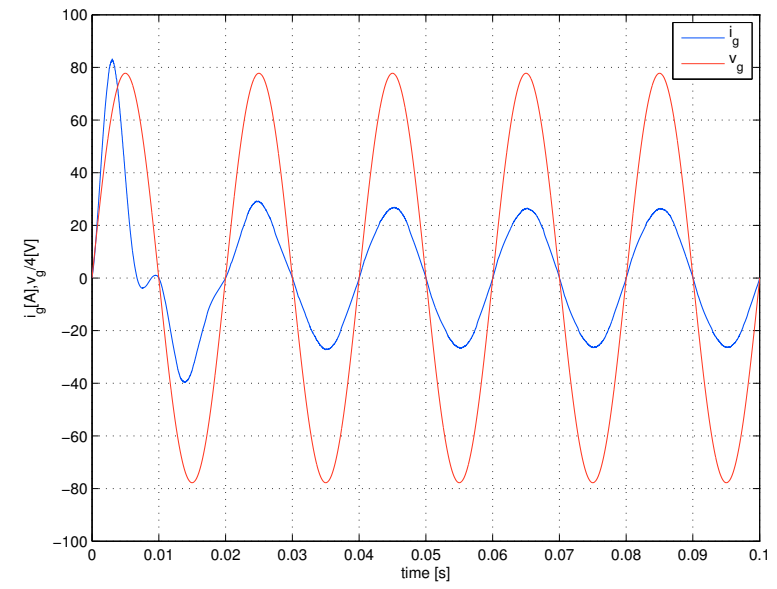

Fig. 8. Simulation results: grid current, $i_{g}$ after a change of the DC bus voltage reference, from $400 \mathrm{~V}$ to $450 \mathrm{~V}$.

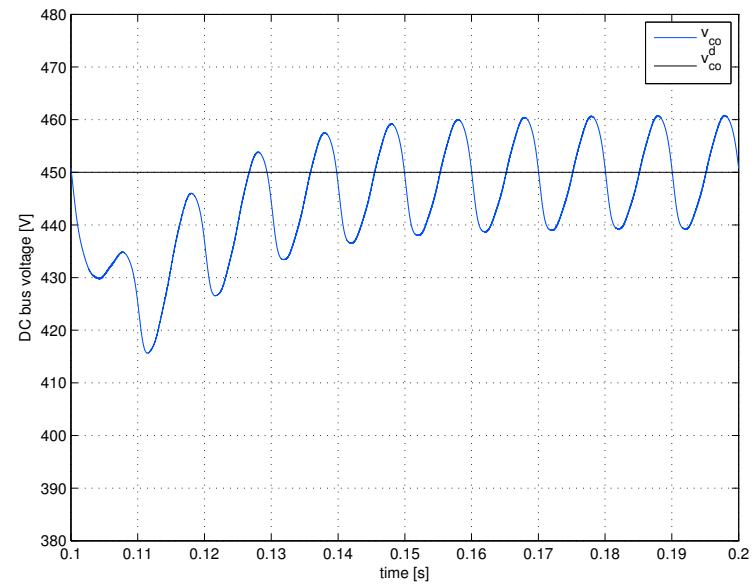

Fig. 9. Simulation results: DC bus voltage after a change of the load value, from $R=50 \Omega$ to $R=10 \Omega$.

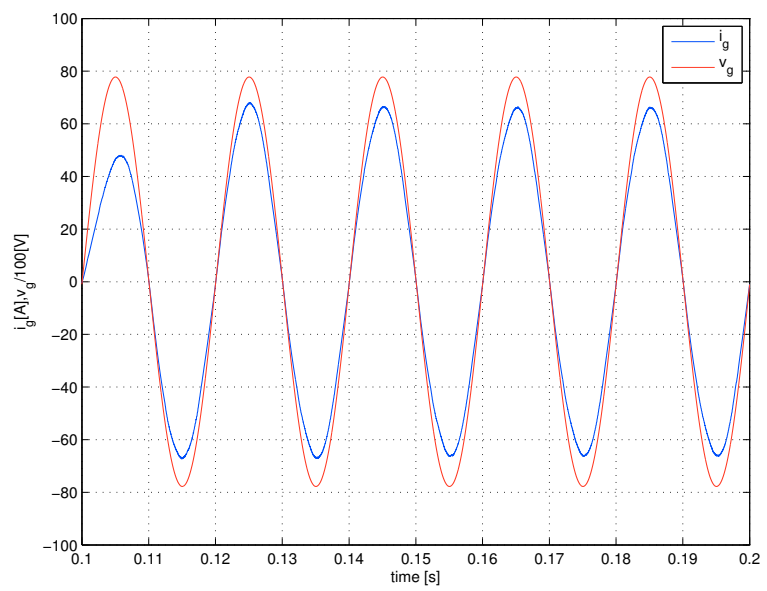

Fig. 10. Simulation results: grid current, $i_{g}$ after a change of the load value, from $R=50 \Omega$ to $R=10 \Omega$.
The feasibility of the combined control strategy was assessed by means of comprehensive numerical simulations conducted on a benchmark model of an existing rectifier, simulating realistic and exacting operating conditions. They demonstrate that the proposed SOSM non-linear approach is a highly efficient solution for the rectifier control problem under study, establishing its viability for implementation in actual single-phase active rectifiers with LCL filter. In accordance with these encouraging results, the following stage of this project will be the refinement and implementation of this SOSM based controller in the laboratory experimental test bench.

\section{ACKNOWLEDGMENT}

This work was supported by the Spanish Government Research Project DPI2010-15110, Project Marie Curie FP72011-IIF-299767/911767 ACRES (EU), CONICET and UNLP (Argentina). The research of C. Kunusch has been supported by the Seventh Framework Programme of the European Community through the Marie Curie actions (GA: PCIG09-GA2011-293876), the Puma-Mind project (GA: FCH-JU-20111-303419), the CICYT project DPI2011-25649 (MINECOSpain) and the CSIC JAE-DOC Research Programme.

\section{REFERENCES}

[1] J. Dannehl, C. Wessels, and F. Fuchs, "Limitations of voltage-oriented PI current control of grid-connected PWM rectifiers with LCL filters," IEEE Trans. on Industrial Electronics, vol. 56, no. 2, pp. 380-388, 2009.

[2] M. Liserre, F. Blaabjerg, and S. Hansen, "Design and control of an LCL-filter-based three-phase active rectifier," IEEE Trans. on Industry Applications, vol. 41, no. 5, pp. 1281-1291, 2005.

[3] Y.-R. Mohamed, M. Rahman, and R. Seethapathy, "Robust line-voltage sensorless control and synchronization of LCL-filtered distributed generation inverters for high power quality grid connection," IEEE Trans. on Power Electronics, vol. 27, no. 1, pp. 87-98, 2012.

[4] E. Wu and P. Lehn, "Digital current control of a voltage source converter with active damping of LCL resonance," IEEE Trans. on Power Electronics, vol. 21, no. 5, pp. 1364-1373, 2006.

[5] J. Dannehl, F. Fuchs, and P. Thogersen, "PI state space current control of grid-connected PWM converters with LCL filters," IEEE Trans. on Power Electronics, vol. 25, no. 9, pp. 2320-2330, 2010.

[6] Y. Tang, P. Loh, P. Wang, F. Choo, and K. Tan, "Improved one-cyclecontrol scheme for three-phase active rectifiers with input inductorcapacitor-inductor filters," IET Power Electronics, vol. 4, no. 5, pp. 603-614, 2011.

[7] V. Utkin, J. Guldner, and J. Shi, Sliding mode control in electromechanical systems. Taylor \& Francis, 1999.

[8] R. Venkataramanan, Sliding mode control of power converters. Dissertation (Ph.D.), California Institute of Technology, 1986.

[9] A. Levant, "Sliding order and sliding accuracy in sliding mode control," International Journal of Control, vol. 58, no. 6, pp. 1247-1263, 1993.

[10] L. Fridman and A. Levant, Sliding Mode Control in Engineering, W. Perruquetti and J. Barbot, Eds. Marcel Dekker Inc., 2002, Chapter 3: Higher Order Sliding Modes, pp. 53-101.

[11] R. Griñó, E. Fossas, and D. Biel, "Sliding mode control of a full-bridge unity power factor rectifier," Lecture Notes in Control and Information Sciences, vol. 281, pp. 139-148, Springer-Berlin, 2002.

[12] E. Cruz-Zavala, J. A. Moreno, and L. Fridman, "Uniform robust exact differentiator," IEEE Trans. on Automatic Control, vol. 56, no. 11, pp. 2727-2733, 2011.

[13] J. Moreno, Lyapunov approach for analysis and design of second order sliding mode algorithms. Springer, 2011. 